H. Fujimoto

Nagoya Math. J.

Vol. 54 (1974) 21-51

\title{
ON FAMILIES OF MEROMORPHIC MAPS INTO THE COMPLEX PROJEGTIVE SPACE
}

\author{
HIROTAKA FUJIMOTO
}

\section{§ 1. Introduction.}

In [10], P. Montel defined the notion of a quasi-normal family of meromorphic functions and obtained several results relating to this. Afterwards, in [13], H. Rutishauser generalized some of them to the case of meromorphic functions of several variables. By definition, a quasinormal family of meromorphic functions on a domain $D$ in $C^{n}$ is a family $\mathscr{F}$ such that any sequence in $\mathscr{F}$ has a subsequence which converges compactly outside a thin analytic subset of $D$. We introduce in this paper the notion of a meromorphically normal family of meromorphic maps into the $N$-dimensional complex projective space $P_{N}(C)$, which is defined as a family $\mathscr{F}$ satisfying the following condition:

Any sequence $\left\{f^{(p)}\right\}$ in $\mathscr{F}$ has a subsequence $\left\{f^{\left(p_{k}\right)}\right\}$ with the property that, on some neighborhood $U$ of each point in $D$, each $f^{\left(p_{k}\right)}$ can be written

$$
f^{\left(p_{k}\right)}=f_{0}^{\left(p_{k}\right)}: f_{1}^{\left(p_{k}\right)}: \cdots: f_{N}^{\left(p_{k}\right)}
$$

for fixed homogeneous coordinates on $P_{N}(C)$ so that $\left\{f_{i}^{\left(p_{k)}\right)}(0 \leqq i \leqq N)\right.$ converges compactly on $U$ to a holomorphic function $f_{i}$ and at least one $f_{i}$ does not vanish identically.

The main purpose of this paper is to give some sufficient conditions for a family of meromorphic maps of a domain $D\left(\subset C^{n}\right)$ into $P_{N}(C)$ to be meromorphically normal.

After some preparatory considerations ( $\$ 2$ and $\S 3$ ), by the full use of the results of W. Stoll in [16] we shall show in $\S 4$ the following improvement of H. Rutishauser's result.

For a family $\mathscr{F}$ of meromorphic maps of $D$ into $P_{N}(C)$, if there exist $2 N+1$ hyperplanes $H_{j}(0 \leqq j \leqq 2 N)$ in $P_{N}(C)$ located in general position

Received August 3, 1973. 
such that $f(D) \not \subset H_{j}(f \in \mathscr{F})$ and for any compact set $K \subset D$ the areas of $f^{-1}\left(H_{j}\right) \cap K$ inclusive of multiplicities are bounded above by a fixed constant, then $\mathscr{F}$ is meromorphically normal (Theorem 4.3).

We can define the characteristic function $T(r, f)(0<r<R)$ of a meromorphic map $f$ defined on a sphere $B(R)$ in $C^{n}$ with center at the origin and radius $R$ and give some elementary properties on the analogy of H. Cartan's work [1]. It will be shown that, for a family $\mathscr{F}, T(r, f)(0<r<R, f \in \mathscr{F})$ are bounded above by a constant depending only on $r$ if and only if $\mathscr{F}$ is meromorphically normal and normal at the origin ( $\S 5)$. The fundamental inequality given in [1] will be also generalized to the case of meromorphic maps of several variables under suitable assumptions (§6). As its consequence, we shall give in $\S 7$ a defect relation for meromorphic maps of several variables and its application.

In the last section, using the fundamental inequality, we shall give the proof of the following theorem, which was firstly stated in the case of $n=1$ by $\mathrm{G}$. Valiron.

Let $\mathscr{F}$ be a family of meromorphic functions on a domain in $\boldsymbol{C}^{n}$ without indetermination points. Suppose that for mutually distinct values $a_{1}$, $a_{2}, \cdots, a_{q}$ any $\varphi(z)-a_{j}(\varphi \in \mathscr{F})$ has no zeros of multiplicity $<m_{j}$ and

$$
\sum_{j=1}^{q} \frac{1}{m_{j}}<q-2 .
$$

Then, $\mathscr{F}$ is a normal family.

For a family of meromorphic maps into $P_{N}(C)$, we shall give also an analogous sufficient condition for $\mathscr{F}$ to be meromorphically normal (Theorem 8.1) and a Schottky-Landau type theorem (Theorem 8.7).

\section{\$2. Preliminaries.}

Let $G$ be a domain in $C^{n}$ and $f$ a not identically zero holomorphic function on $G$. For a point $a=\left(a_{1}, a_{2}, \cdots, a_{n}\right) \in G$ we expand $f$ as a compactly convergent series

$$
f\left(u_{1}+a_{1}, \cdots, u_{n}+a_{n}\right)=\sum_{m=0}^{\infty} P_{m}\left(u_{1}, \cdots u_{n}\right)
$$

on a neighborhood of $a$, where $P_{m}$ is either identically zero or a homogeneous polynomial of degree $m$. The number 


$$
\nu_{f}(a):=\min \left\{m ; P_{m}(u) \not \equiv 0\right\}
$$

is said to be the zero multiplicity of $f$ at $a$. By definition, a divisor on $G$ is an integer-valued function $\nu$ on $G$ such that for every $a \in G$ there are holomorphic functions $g(z)(\not \equiv 0)$ and $h(z)(\not \equiv 0)$ on a neighborhood $U$ of $a$ with $\nu(z)=\nu_{g}(z)-\nu_{h}(z)$ on $U$. The carrier of a divisor $\nu$ is defined as an analytic set

$$
|\nu|:=\overline{\{z \in G ; \nu(z) \neq 0\}}
$$

in $G$. As is easily seen, for a holomorphic function $f(\not \equiv 0)$ on $G, \nu_{f}(z)$ equals the zero multiplicity of $f$ at $z$ in the sence of Definition 2.1 in [7] whenever $z$ is a regular point of $|\nu|$.

Let us consider next a meromorphic maps $f$ of $G$ into $P_{N}(C)$. For any $a \in G, f$ has a representation

$$
f(z)=f_{0}(z): f_{1}(z): \cdots: f_{N}(z)
$$

on some neighborhood $U$ of $a$ with fixed homogeneous coordinates $w_{0}: w_{1}: \cdots: w_{N}$ on $P_{N}(C)$ and holomorphic functions $f_{i}(z)(0 \leqq i \leqq N)$ on $U$, where we can choose them so as to satisfy the condition

$$
\operatorname{codim}\left\{f_{0}(z)=f_{1}(z)=\cdots=f_{N}(z)=0\right\} \geqq 2 .
$$

A representation of $f$ satisfying this condition is referred to as an admissible representation of $f$ on $U$ in the following sections.

Take a hyperplane $H$ in $P_{N}(C)$ with $f(G) \not \subset H$ defined by

$$
H: a^{0} w_{0}+a^{1} w_{1}+\cdots+a^{N} w_{N}=0 .
$$

For every $a \in G$, taking an admissible representation $f=f_{0}: f_{1}: \cdots: f_{N}$ on a neighborhood $U$ of $a$, we consider a holomorphic function

$$
F:=a^{0} f_{0}+a^{1} f_{1}+\cdots+a^{N} f_{N} .
$$

Then, the divisor $\nu(f, H)(z):=\nu_{F}(z)(z \in U)$ is determined independently of a choice of admissible representations and hence is well-defined on the totality of $G$. A meromorphic function $\varphi$ on $G$ induces a meromorphic map $\varphi^{*}$ of $G$ into $P_{1}(C)$ defined by $\varphi^{*}(z):=f_{0}(z): f_{1}(z)$ on a connected open set $U$ if $\varphi=f_{0}(z) / f_{1}(z)$ for holomorphic functions $f_{0}, f_{1}(\not \equiv 0)$ on $U$. In this case, $\nu_{\varphi}^{0}:=\nu\left(\varphi^{*}, H_{0}\right)$ and $\nu_{\varphi}^{\infty}:=\nu\left(\varphi^{*}, H_{1}\right)$ are nothing but the divisors of zeros of $\varphi$ and of poles of $\varphi$ respectively, where $H_{i}=\left\{w_{i}=0\right\}$ $(i=0,1)$. 
Let $\nu$ be a non-negative divisor on a sphere $B(R):=\{\|z\|<R\}$ $(0<R \leqq+\infty)$, where for $z=\left(z_{1}, z_{2}, \cdots, z_{n}\right)$

$$
\|z\|:=\left(\left|z_{1}\right|^{2}+\left|z_{2}\right|^{2}+\cdots+\left|z_{n}\right|^{2}\right)^{\frac{1}{2}} .
$$

DEFinition 2.1. Suppose that $0<m \leqq+\infty$ and $0<s<r<R$. We define

$$
\begin{aligned}
V_{m}(r, \nu): & =\int_{|\nu| \cap \overline{B(r)}} \min (\nu(z), m) v_{n-1}(z) & & \text { in case of } n>1 \\
& =\sum_{|z| \leqq r} \min (\nu(z), m) & & \text { in case of } n=1
\end{aligned}
$$

and

$$
N_{m}(r, s ; \nu):=\frac{1}{W} \int_{s}^{r} \frac{V_{m}(r, \nu)}{t^{2 n-1}} d t
$$

where $v_{1}=(i / 2) \sum_{k=1}^{n} d z_{k} \wedge d \bar{z}_{k}, v_{n-1}=(1 /(n-1) !) v_{1} \wedge v_{1} \wedge \cdots \wedge v_{1}((n-1)-$ times), $W:=\left(\pi^{n-1} /(n-1) !\right)$ and the integral over $|\nu| \cap \overline{B(r)}$ means the integral over the manifold consisting of all regular points of $|\nu| \cap \overline{B(r)}$. And, we put $V(r, \nu)=V_{\infty}(r, \nu), N(r, s ; \nu)=N_{\infty}(r, s ; \nu)$ and $N_{m}(r, \nu):=$ $\lim _{s \rightarrow+0} N_{m}(r, s ; \nu)$ for the case $0 \notin|\nu|$.

Since $B(R)$ is a Cousin-II domain, any given divisor $\nu$ on $B(R)$ can be written

$$
\nu=\nu_{g}-\nu_{h}
$$

with holomorphic functions $g(\not \equiv 0)$ and $h(\not \equiv 0)$ on $B(R)$. Consider holomorphic functions $g_{a}^{\sharp}(u)=g(a u)$ and $h_{a}^{\sharp}(u)=h(a u)$ of $u$ for each $a=$ $\left(a_{1}, a_{2}, \cdots, a_{n}\right) \in S(1):=\{\|z\|=1\}$, where $a u=\left(a_{1} u, a_{2} u, \cdots, a_{n} u\right)$. The set

$$
E:=\left\{a \in S(1) ; g_{a}^{\sharp}(u) \equiv 0 \text { or } h_{a}^{\sharp}(u) \equiv 0\right\}
$$

is obviously a set of measure zero in $S(1)$. Define

$$
\nu[a](u):=\nu_{g_{a}^{\sharp}}(u)-\nu_{h_{a}^{\sharp}}(u)
$$

for each $a \in S(1)-E$. We have then

(2.2) There exists a subset $F(\supseteq E)$ of measure zero in $S(1)$ such that $\nu[a](u)=\nu(a u)(0<|u|<R)$ for any $a \in S(1)-F$ (c.f., [7], Proposition 2.7).

Following W. Stoll [16], we put $\Phi(r):=\left(2 \pi^{n} /(n-1) !\right) r^{2 n-1}$ and denote by $\sigma_{r}$ the Euclidean volume element of $S(r):=\{\|z\|=r\}$. As was shown in [16], pp. 162-164, it holds that 


$$
V_{m}(r, \nu)=\frac{r^{2 n-2}}{2 \pi} \int_{a \in S(1)} V_{m}(r, \nu[a]) \sigma_{1}(a)
$$

and

$$
N_{m}(r, s ; \nu)=\frac{1}{\Phi(1)} \int_{a \in S(1)} N_{m}(r, s ; \nu[a]) \sigma_{1}(a) .
$$

We have also the following Jensen's formula ([16], Proposition 1.6).

(2.4) Let $\varphi(\not \equiv 0)$ be a meromorphic function on $B(R)$. Then

$$
N\left(r, s ; \nu_{\varphi}^{0}\right)=N\left(r, s ; \nu_{\varphi}^{\infty}\right)+\frac{1}{\Phi(r)} \int_{S(r)} \log |\varphi| \sigma_{r}-\frac{1}{\Phi(s)} \int_{S(s)} \log |\varphi| \sigma_{s},
$$

where $0<s<r<R$. For the case $0 \notin\left|\nu_{\varphi}^{0}\right| \cup\left|\nu_{\varphi}^{\infty}\right|$

$$
N\left(r, \nu_{\varphi}^{0}\right)=N\left(r, \nu_{\varphi}^{\infty}\right)+\frac{1}{\Phi(r)} \int_{S(r)} \log |\varphi| \sigma_{r}-\log |\varphi(0)| .
$$

DEFINITION 2.5 (c.f., [16], p. 176). Let $\left\{\nu_{\lambda} ; \lambda \in \Lambda\right\}$ be a directed set of non-negative divisors on a domain $G\left(\subset C^{n}\right)$. It is said to converge to a non-negative divisor $\nu$ on $G$ if and only if any $a \in G$ has a neighborhood $U$ such that, for suitable holomorphic functions $h^{(\lambda)}(\not \equiv 0)$ and $h(\not \equiv 0)$ on $U, \nu_{\lambda}=\nu_{h(\lambda)}, \nu=\nu_{h}$ and $\left\{h^{(\lambda)}: \lambda \in \Lambda\right\}$ converges compactly to $h$ on $U$.

The space $\mathscr{D}^{+}(G)$ of all non-negative divisors on $G$ has a Hausdorff topology which is compatible with this notion of convergence.

In the following sections, we need

(2.6) A subset $\mathscr{N}$ of $\mathscr{D}^{+}(B(R))$ is relatively compact if and only if $V(r, \nu)(0<r<R, \nu \in \mathscr{N})$ are bounded above by a constant depending only on $r$ ([16], Theorem 2.24).

(2.7) Let $H(B(r))$ be the space of all holomorphic functions on $B(r)$ endowed with the compact convergence topology, where $0<r<R$. Then, there exists a continuous map.

$$
\gamma: \mathscr{D}_{0}^{+}(B(R)):=\left\{\nu \in \mathscr{D}^{+}(B(R)) ; \nu(0)=0\right\} \rightarrow H(B(r))
$$

$(0<r<R)$ such that $\nu=\nu_{r_{(\nu)}}$ for any $\nu \in \mathscr{D}_{0}^{+}(B(R))$ ([16], Theorem 3.6).

(2.8) If a sequence $\left\{\nu_{p}\right\}$ converges to $\nu$ in $\mathscr{D}^{+}(B(R))$, then $\left\{\left|\nu_{p}\right|\right\}$ converges to $|\nu|$ in the sense that $|\nu|$ coincides with the set of all $z$ such that every neighborhood $U$ of $z$ intersects $\left|\nu_{p}\right|$ for all but finitely many $p$ and, 
simultaneously, with the set of all $z$ such that every $U$ intersects $\left|\nu_{p}\right|$ for infinitely many $p$ ([16], Theorem 4.10).

Let $S$ be an analytic set of codimension $\geqq 2$ in $G$. By the well-known Thullen-Remmert-Stein's theorem (c.f., [17]), any divisor $\nu \in \mathscr{D}^{+}(G-S$ ) can be uniquely extended to $\hat{\nu} \in \mathscr{D}^{+}(G)$. Moreover, we have

(2.9) If $\left\{\nu_{p}: p=1,2, \cdots\right\}$ in $\mathscr{D}^{+}(G-S)$ converges to $\nu$ on $G-S$, then $\left\{\hat{\nu}_{p}\right\}$ converges to $\hat{\nu}$ in $\mathscr{D}^{+}(G)$, where $\hat{\nu}_{p}$ and $\hat{\nu}$ are the extensions of $\nu_{p}$ and $\nu$ to $G$ respectively.

In fact, according to [11], Theorem II, $\left\{\hat{\nu}_{p}\right\}$ is normal i.e., relatively compact in $\mathscr{D}^{+}(G)$ because any pseudoconvex domain which includes $G-S$ includes necessarily the totality of $G$. On the other hand, the limit of any convergent subsequence of $\left\{\hat{\nu}_{p}\right\}$ is obviously equal to $\hat{\nu}$. Therefore, $\left\{\hat{\nu}_{p}\right\}$ itself converges to $\hat{\nu}$ on $G$.

\section{$\S 3$. Definition and some properties of $m$-convergent sequences.}

Let $\left\{f^{(p)} ; p=1,2, \cdots\right\}$ be a sequence of meromorphic maps of a domain $G\left(\subset C^{n}\right)$ into $P_{N}(C)$.

DEFINITION 3.1. We shall call $\left\{f^{(p)}\right\}$ to converge meromorphically (or simply $m$-converge) on $G$ to a meromorphic map $f$ if and only if, for any $a \in G$, each $f^{(p)}$ has an admissible representation

$$
f^{(p)}(z):=f_{0}^{(p)}(z): f_{1}^{(p)}(z): \cdots: f_{N}^{(p)}(z)
$$

on some neighborhood $U$ of $a$ such that $\left\{f_{i}^{(p)}\right\}(0 \leqq i \leqq N)$ converge compactly to holomorphic functions $f_{i}(z)$ with the property

$$
f(z)=f_{0}(z): f_{1}(z): \cdots: f_{N}(z)
$$

on $U$, where $f_{i_{0}}(z) \not \equiv 0$ for some $i_{0}$.

We note first the following fact.

(3.2) Consider another system of admissible representations

$$
f^{(p)}=g_{0}^{(p)}: g_{1}^{(p)}: \cdots: g_{N}^{(p)}
$$

of each $f^{(p)}$ on $U$ such that $\lim _{p \rightarrow \infty} g_{i}^{(p)}=g_{i}$ exists for any $i$ and $g_{j_{0}} \not \equiv 0$ for some $j_{0}$. Then, there is a nowhere zero holomorphic function $h$ on $U$ such that 


$$
g_{i}(z)=h(z) f_{i}(z) \quad(z \in U)
$$

for any $i(0 \leqq i \leqq N)$.

In fact, by the definition of admissible representations, we can describe $g_{i}^{(p)}=h^{(p)} f_{i}^{(p)}(0 \leqq i \leqq N)$ with a nowhere zero holomorphic function $h^{(p)}$. Then, $h(z)=\lim _{p \rightarrow \infty} h^{(p)}(z)$ exists evidently on $U-\left\{f_{i_{0}}=0\right\}$ and, hence, on the totality of $U$ by the maximum principle. According to the classical Hurwitz theorem, we have then either $h \equiv 0$ or $h \neq 0$ everywhere. On the other hand, the assumption $g_{j_{0}} \neq \equiv 0$ implies that $h \not \equiv 0$. This leads to (3.2).

For a meromorphic map $f$ into $P_{N}(C)$ we denote by $I(f)$ the set of all points of indetermination of $f$, which is given by the condition

$$
I(f) \cap U=\left\{z \in U: f_{0}(z)=f_{1}(z)=\cdots=f_{N}(z)=0\right\}
$$

if $f$ has an admissible representation $f=f_{0}: f_{1}: \cdots: f_{N}$ on an open subset $U$ of $G$. So, we have $\operatorname{codim} I(f) \geqq 2$.

Now, following H. Rutishauser ([13]), we give

Definition 3.3. A sequence $\left\{f^{(p)}\right\}$ of meromorphic maps of $G$ into $P_{N}(C)$ is said to be quasi-regular if any $a \in G$ has a neighborhood $U$ with the property that $\left\{f^{(p)}\right\}$ converges compactly on $U$ outside a thin analytic subset $S$ of $U$, i.e., for any domain $D$ with $D \in U-S$ there is some $p_{0}$ such that $I\left(f^{(p)}\right) \cap D=\phi\left(p \geqq p_{0}\right)$ and $\left\{f^{(p)} \mid D ; p \geqq p_{0}\right\}$ converges compactly to a holomorphic map of $D$ into $P_{N}(C)$. And, we shall call $\left\{f^{(p)}\right\}$ to be regular at $a \in G$ if $\left\{f^{(p)}\right\}$ converges compactly on some neighborhood of $a$.

Let $\left\{f^{(p)}\right\}$ be an $m$-convergent sequence of meromorphic maps defined on $G$ whose limit $f$ has representations

$$
f=f_{0 \ell}: f_{1 \ell}: \cdots: f_{N \ell}
$$

on open sets $U_{\ell}$ satisfying the condition in Definition 3.1, where $G=$ $\bigcup_{\ell} U_{\ell}$. By (3.2) we can define an analytic set $S$ with

$$
S \cap U_{\ell}=\left\{z \in U_{\ell} ; f_{0 \ell}(z)=\cdots=f_{N \ell}(z)=0\right\}
$$

for any $\ell$. Obviously, $\left\{f^{(p)}\right\}$ converges compactly on $G-S$. So, we see

(3.4) An m-convergent sequence is always quasi-regular. 
Take a hyperplane

$$
H: a^{0} w_{0}+a^{1} w_{1}+\cdots+a^{N} w_{N}=0
$$

in $P_{N}(C)$ with $f(G) \not \subset H$. Put

$$
F_{\ell}:=a^{0} f_{0 \ell}+a^{1} f_{1 \ell}+\cdots+a^{N} f_{N \ell}
$$

on $U_{\ell}$ and define a divisor $\nu^{H}$ on $G$ by $\nu^{H}=\nu_{F_{\ell}}$ on each $U_{\ell}$, which is well-defined by (3.2). Then, $\left\{\nu\left(f^{(p)}, H\right)\right\}$ converges to $\nu^{H}$ in the sense of Definition 2.5.

For example, a sequence

$$
\varphi^{(p)}(z)=\frac{1}{z^{p}}\left(z+\frac{1}{p^{2}}\right)^{p} \quad(p=1,2 \cdots)
$$

is not $m$-convergent on $C$ because $\left\{\nu_{\varphi(p)}^{0}\right\}$ has no limit, though it is quasiregular and the limit is of constant.

Proposition 3.5. Let $\left\{f^{(p)}\right\}$ converge to a meromorphic map $f$ on $G$ excluding a thin analytic set $S$. If there exists a hyperplane $H$ such that $f(G-S) \not \subset H$ and $\left\{\nu\left(f^{(p)}, H\right)\right\}$ converges in $\mathscr{D}^{+}(G)$, then $\left\{f^{(p)}\right\}$ is $m$ convergent.

Proof. Without loss of generality, we may assume that $H=\left\{w_{0}=0\right\}$, $G=B(R), 0 \notin S$ and $\left\{f^{(p)}\right\}$ converges compactly on $B(R)-S$ to a holomorphic map $f$ with $f(0) \notin H$. By (2.7), for any $r(0<r<R)$, we can choose holomorphic functions $h^{(p)}(\not \equiv 0)$ and $h(\not \equiv 0)$ on $B(r)$ such that $\nu\left(f^{(p)}, H\right)=\nu_{h(p)}, \nu=\nu_{h}$ for the limit $\nu$ of $\left\{\nu\left(f^{(p)}, H\right)\right\}$ and $\left\{h^{(p)}\right\}$ converges compactly to $h$ on $B(r)$. Then, each $f^{(p)}$ has an admissible representation

$$
f^{(p)}=h^{(p)}: f_{1}^{(p)}: \cdots: f_{N}^{(p)}
$$

with suitable holomorphic functions $f_{i}^{(p)}(0 \leqq i \leqq N)$ on $B(r)$. For our purpose, it suffices to show that $\left\{f_{i}^{(p)}\right\}$ converges compactly on $B(r)$. The problem is of local. For any $a \in B(r)$, take neighborhoods $U$ and $V$ of $a$ such that $V \subset U \subset B(r),(\bar{U}-V) \cap(S \cup\{h=0\})=\phi$ and

$$
\sup \{|\varphi(z)| ; z \in \bar{U}-V\}=\sup \{|\varphi(z)| ; z \in \bar{U}\}
$$

for any holomorphic function $\varphi(z)$ on a neighborhood of $\bar{U}$. It may be assumed that $h^{(p)}(p=1,2, \cdots)$ has no zeros on $U-V$. We have thus representations 


$$
f^{(p)}=1: \frac{f_{1}^{(p)}}{h^{(p)}}: \cdots: \frac{f_{N}^{(p)}}{h^{(p)}}
$$

and, moreover,

$$
f=1: \varphi_{1}: \cdots: \varphi_{N}
$$

with holomorphic functions $\varphi_{i}:=\lim _{p \rightarrow \infty}\left(f_{i}^{(p)} / h^{(p)}\right)$ on $U-V$ because $\lim _{p \rightarrow \infty} f^{(p)}=f$ on $U-V$. On the other hand, since $f_{i}^{(p)}=h^{(p)}\left(f_{i}^{(p)} / h^{(p)}\right)$, $\left\{f_{i}^{(p)}\right\}$ converges compactly on $U-V$. Then, by the use of (3.6), we can easily conclude that $\left\{f_{i}^{(p)}\right\}$ converges compactly on the totality of $U$. This completes the proof.

COROLLARY 3.7. If a sequence $\left\{f^{(p)}\right\}$ is regular at every point in a domain $G$ except an analytic set $S$ of codimension $\geqq 2$, then $\left\{f^{(p)}\right\}$ is $m$ convergent on the totality of $G$.

Proof. Put $f:=\lim _{p \rightarrow \infty} f^{(p)}$ on $G-S$ and take a hyperplane $H$ in $P_{N}(C)$ with $f(G-S) \varangle H$. Then, $\left\{\nu\left(f^{(p)}, H\right)\right\}$ converges to $\nu(f, H)$ on $G$ $-S$. By the assumption and (2.9), it converges also on the totality of G. Proposition 3.5 gives Corollary 3.7 directly.

For later use, we give here

Proposition 3.8. Let $\left\{f^{(p)}\right\}$ be an m-convergent sequence of meromorphic maps of $B(R)$ into $P_{N}(C)$ with the limit $f$. Then, for any $r$ $(0<r<R)$, each $f^{(p)}$ has an admissible representation

$$
f^{(p)}=f_{0}^{(p)}: f_{1}^{(p)}: \cdots: f_{N}^{(p)}
$$

on $B(r)$ such that $\left\{f_{i}^{(p)}\right\}$ converge compactly to holomorphic functions $f_{i}$ on $B(r)$ satisfying the condition

$$
f=f_{0}: f_{1}: \cdots: f_{N},
$$

where $f_{i_{0}}(z) \not \equiv 0$ for some $i_{0}$.

Proof. By Definition 3.1, $\overline{B(r)}$ can be covered by finitely many open sets $U_{\ell}$ such that each $f^{(p)}$ has admissible representations

$$
f^{(p)}=f_{0 \ell}^{(p)}: f_{1 \ell}^{(p)}: \cdots: f_{N \ell}^{(p)}
$$

on $U_{\ell}$ and $\lim _{p \rightarrow \infty} f_{i \ell}^{(p)}=f_{i \ell}$ exist, where we may assume $f_{0 \ell}(0) \neq 0, f_{0 \ell}^{(p)}(0)$ $\neq 0$ for any $\ell$ and $p$. Then, $\left\{\nu\left(f^{(p)}, H_{0}\right)\right\}$ converges to a divisor $\nu\left(=\nu_{f_{0 \ell}}\right.$ on each $U_{\ell}$ ), where $H_{0}=\left\{w_{0}=0\right\}$. By (2.7), there are holomorphic 
functions $h^{(p)}$ and $h$ on $B(r)$ such that $\nu_{h(p)}=\nu\left(f^{(p)}, H_{0}\right), \nu_{h}=\nu_{0}$ and $\left\{h^{(p)}\right\}$ converges compactly to $h$ on $B(r)$. Since $h^{(p)} / f_{0 \ell}^{(p)}$ are nowhere zero holomorphic functions and $f_{i \ell}^{(p)} / f_{0 \ell}^{(p)}=f_{i m}^{(p)} / f_{0 m}^{(p)}$ on $U_{\ell} \cap U_{m}$, we obtain admissible representations

$$
f^{(p)}=f_{0}^{(p)}: f_{1}^{(p)}: \cdots: f_{N}^{(p)}
$$

on the totality of $B(r)$ with holomorphic functions

$$
f_{0}^{(p)}:=h^{(p)}, \quad f_{1}^{(p)}:=\frac{h^{(p)}}{f_{0 \ell}^{(p)}} f_{1 \ell}^{(p)}, \cdots, f_{N}^{(p)}:=\frac{h^{(p)}}{f_{0 \ell}^{(p)}} f_{N \ell}^{(p)}
$$

on each $U_{\ell}$. On the other hand, $\left\{h^{(p)} / f^{(p)}\right\}$ converges compactly to $h / f_{0 \ell}$ on $U_{\ell}-\left\{f_{0 \ell}=0\right\}$ and, hence, on the totality of $U_{\ell}$ to a holomorphic function $h / f_{0 \ell}$ by the maximum principle. Therefore, $\left\{f_{i}^{(p)}\right\}(0 \leqq i \leqq N)$ converges on $B(r)$ to holomorphic functions $f_{i}:=\left(h / f_{0 \ell}\right) f_{i \ell}(0 \leqq i \leqq N)$, which satisfy the condition $f=f_{0}: f_{1}: \cdots: f_{N}$. This completes the proof.

\section{§ 4. An improvement of H. Rutishauser's result.}

Let us consider a family $\mathscr{F}$ of meromorphic maps of a domain $G$ in $C^{n}$ into $P_{N}(C)$.

DEFINITION 4.1. We shall call $\mathscr{F}$ to be meromorphically normal (or simply $m$-normal) if any sequence in $\mathscr{F}$ has an $m$-convergent subsequence and to be normal at $a(\in G)$ if any sequence in $\mathscr{F}$ has a subsequence which converges compactly on some neighborhood of $a$, maybe, depending on each sequence.

The definition of $m$-normalcy coincides with the definition given in $\S 1$. Because, we can assert

(4.2) Let $\left\{f^{(p)}\right\}$ be a sequence of meromorphic maps of $B(R)$ into $P_{N}(C)$. Suppose that each $f^{(p)}$ has a (not necessarily admissible) representation

$$
f^{(p)}=f_{0}^{(p)}: f_{1}^{(p)}: \cdots: f_{N}^{(p)}
$$

such that $\left\{f_{i}^{(p)}\right\}$ converge compactly to holomorphic functions $f_{i}$ on $B(R)$, where $f_{i_{0}} \equiv \equiv$ for some $i_{0}$. Then, $\left\{f^{(p)}\right\}$ has an m-convergent subsequence.

To see this, take a hyperplane

$$
H: a^{0} w_{0}+a^{1} w_{1}+\cdots+a^{N} w_{N}=0
$$


with $f(B(R)) \not \subset H$, where $f:=f_{0}: \cdots: f_{N}$. Since $\left\{f^{(p)}\right\}$ is obviously quasiregular, by Proposition 3.5 we have only to show that $\left\{\nu\left(f^{(p)}, H\right)\right\}$ has a convergent subsequence. Consider holomorphic functions

$$
F^{(p)}:=a^{0} f_{0}^{(p)}+a^{1} f_{1}^{(p)}+\cdots+a^{N} f_{N}^{(p)},
$$

Then, since $\left\{\nu_{F^{(p)}}\right\}$ converges, by (2.6) $V\left(r, \nu_{F^{(}(p)}\right)$ are uniformly bounded for any fixed $r(0<r<R)$. On the other hand, $\nu\left(f^{(p)}, H\right) \leqq \nu_{F^{(p)}}$ on $B(R)$ and so

$$
V\left(r, \nu\left(f^{(p)}, H\right)\right) \leqq V\left(r, \nu_{F(p)}\right)
$$

$(0<r<R)$. Therefore, $V\left(r, \nu\left(f^{(p)}, H\right)\right)$ are also uniformly bounded. By (2.6), we obtain the desired conclusion.

We give now the following improvement of H. Rutishauser's result.

THEOREM 4.3. Let $\mathscr{F}$ be a family of meromorphic maps of $B(R)$ into $P_{N}(C)$ and $H_{j}(0 \leqq j \leqq 2 N)$ be $2 N+1$ hyperplanes in $P_{N}(C)$ located in general position such that for any $f \in \mathscr{F} f(B(R)) \not \subset H_{j}$. If $V\left(r, \nu\left(f, H_{j}\right)\right)$ $(0 \leqq j \leqq 2 N)$ are uniformly bounded for any fixed $r(0<r<R)$, then $\mathscr{F}$ is m-normal.

Proof. Take an arbitrary sequence $\left\{f^{(p)}\right\}$ in $\mathscr{F}$. By the assumption and (2.6), choosing a subsequence and changing indices if necessary, we may assume that $\left\{\nu\left(f^{(p)}, H_{j}\right)\right\}$ converges to a non-negative divisor $\nu_{j}$ on $B(R)(0 \leqq j \leqq 2 N)$. Then, each $\left\{\left|\nu\left(f^{(p)}, H_{j}\right)\right| ; p=1,2, \cdots\right\}$ converges to $\left|\nu_{j}\right|$ as a sequence of closed subsets of $B(R)$. We put $S:=\bigcup_{j=0}^{2 N}\left|\nu_{j}\right|$, which is a thin analytic subset of $B(R)$. Let $D$ be an arbitrary domain with $D \subset B(R)-S$. Since $I\left(f^{(p)}\right) \subset\left|\nu\left(f^{(p)}, H_{j}\right)\right|$ for any $p$ and every $a \in B(R)$ - $S$ has a neighborhood $U$ such that $U \cap\left|\nu\left(f^{(p)}, H_{j}\right)\right|=\phi$ for all but finitely many $p$, taking a sufficientlly large $p_{0}$, we see that every $f^{(p)}$ is holomorphic on $D$ and

$$
f^{(p)}(D) \subset P_{N}(C)-\bigcup_{j=0}^{2 N} H_{j}
$$

for any $p \geqq p_{0}$. Then, $\left\{f^{(p)} \mid D\right\}$ has a compactly convergent subsequence as a sequence of holomorphic maps of $D$ into $P_{N}(C)$ because of J. Dufresnoy's theorem (c.f., [3], Critère fondamental [6] and [9], Corollary 3). Therefore, by the usual diagonal argument, we can find a subsequence of $\left\{f^{(p)}\right\}$ which converges on $B(R)-S$ in the sense of Definition 3.3. From the beginning, $\left\{f^{(p)}\right\}$ itself may be assumed to converge to a holomorphic 
map $f$ of $B(R)-S$ into $P_{N}(C)$. We can take here some $j_{0}$ with $f(B(R)$ $-S) \not \subset H_{j_{0}}$ because $H_{j}(0 \leqq j \leqq 2 N)$ are located in general position. On the other hand, $\left\{\nu\left(f^{(p)}, H_{j_{0}}\right)\right\}$ is convergent in $\mathscr{D}^{+}(B(R))$. Thus, the assumptions of Proposition 3.5 are satisfied. We can conclude that $\left\{f^{(p)}\right\}$ is $m$-convergent on $B(R)$. The proof is complete.

COROLlaRY 4.4. In the same situation as in Theorem 4.3, if, for any complex line $\ell$ in $B(R)$ through the origin, either $\ell \subset f^{-1}\left(H_{j}\right)$ or the number of elements in $\ell \cap f^{-1}\left(H_{j}\right)$ counted with multiplicities are bounded above by a constant $q_{j}$ not depending on each $\ell$ and each $f \in \mathscr{F}$, then $\mathscr{F}$ is m-normal.

Proof. For any fixed $f \in \mathscr{F}$, take an arbitrary $a \in S(1)$ such that $\ell:=\{a u ;|u|<R\} \not \subset\left|\nu\left(f, H_{j}\right)\right|$. By the assumption, we have

$$
V\left(r, \nu\left(f, H_{j}\right)[a]\right) \leqq q_{j}
$$

$(0 \leqq j \leqq 2 N, 0<r<R)$. By (2.3) we conclude

$$
V\left(r, \nu\left(f, H_{j}\right)\right) \leqq q_{j} W r^{2 n-2} .
$$

This gives Corollary 4.4 as a result of Theorem 4.3.

Remark. H. Rutishauser proved that, under the assumption of Theorem 4.3 or of Corollary 4.4, any sequence in $\mathscr{F}$ has a quasi-regular subsequence in the case $n=2$ and $N=1$ (c.f., [13], Satz 18).

CoRollary 4.5. For $\mathscr{F}$ and $H_{j}$ with the property as in Theorem 4.3 and an arbitrarily fixed $s(0<s<R)$, if $N\left(r, s ; \nu\left(f, H_{j}\right)\right.$ ) are bounded by a constant depending only on $r(s<r<R)$, then $\mathscr{F}$ is m-normal.

Proof. This is a consequence of the inequalities

$$
\begin{aligned}
N\left(\rho, s ; \nu\left(f, H_{j}\right)\right) & \geqq \int_{r}^{\rho} \frac{V\left(t, \nu\left(f, H_{j}\right)\right)}{W t^{2 n-1}} d t \\
& \geqq c(\rho, r) V\left(r, \nu\left(f, H_{j}\right)\right),
\end{aligned}
$$

where $\rho(r<\rho<R)$ is arbitrarily fixed and

$$
c(\rho, r):=\int_{r}^{\rho} \frac{d t}{W t^{2 n-1}}(>0) .
$$

§5. Characteristic functions for meromorphic maps into $P_{N}(C)$.

We shall give first the definition and elementary properties of the 
characteristic functions of meromorphic maps of $B(R)$ into $P_{N}(C)$ along the lines of H. Cartan's work [1]. For brevity, we assume all meromorphic maps treated in the following sections to be holomorphic at the origin unless otherwise stated.

Let $f$ be a meromorphic map of $B(R)$ into $P_{N}(C)$ with an admissible representation $f=f_{0}: f_{1}: \cdots: f_{N}$ on $B(R)$. We put

$$
u(z):=\max _{0 \leqq i \leqq N} \log \left|f_{i}(z)\right| .
$$

DEFINITION 5.1. The characteristic function is defined by

$$
T(r, f):=\frac{1}{\Phi(r)} \int_{S(r)} u(z) \sigma_{r}-u(0)
$$

Remark. (i) $T(r, f)$ is monotone increasing and convex as a function of $\log r$, because $u(z)$ is plurisubharmonic.

(ii) In [14], W. Stoll called the function

$$
\tilde{T}(r):=\frac{1}{\Phi(r)} \int_{S(r)} \log \|f\| \sigma_{r}-\frac{1}{\Phi(s)} \int_{S(s)} \log \|f\| \sigma_{s}
$$

the characteristic function of $f$, where $0 \leqq s<r<R$ and $\|f\|^{2}=\left|f_{0}\right|^{2}+$ $\left|f_{1}\right|^{2}+\cdots+\left|f_{N}\right|^{2}$ for an admissible representation $f=f_{0}: f_{1}: \cdots: f_{N}$. As is easily seen,

$$
|T(r, f)-\tilde{T}(r)| \leqq K
$$

for some constant $K$.

(5.2) $T(r, f)$ is uniquely determined independently of a choice of admissible representations of $f$.

In fact, this can be proved in the same manner as in [1], p. 8 by the use of (2.4).

Let $E$ be the set of all points $a$ in $S(1)$ such that $\{u a ;|u|<R\} \subset I(f)$, which is of measure zero in $S(1)$. For any $a \in S(1)-E$ consider a meromorphic map $f_{a}^{\sharp}(u):=f(a u)$ of $\{u ;|u|<R\}(\subset C)$ into $P_{N}(C)$. Since

$$
T\left(r, f_{a}^{\#}\right)=\frac{1}{2 \pi} \int_{0}^{2 \pi} u\left(a r e^{i \theta}\right) d \theta-u(0),
$$

for any $a \in S(1)-E$, we have by Lemma 1.1 and Lemma 1.2 in [16] 


$$
T(r, f)=\frac{1}{\Phi(1)} \int_{a \in S(1)} T\left(r, f_{a}^{\#}\right) \sigma_{1}(a)
$$

Take a non-singular matrix $\left(\left(a_{j}^{i} ; 0 \leqq i, j \leqq N\right)\right)$ and consider a meromorphic map $g=g_{0}: g_{1}: \cdots: g_{N}$ defined by holomorphic functions $g_{j}=$ $\sum_{i=1}^{N} a_{j}^{i} f_{i}$. Then,

$$
|T(r, f)-T(r, g)| \leqq K
$$

for some constant $K$ depending only on $a_{j}^{i}$ (c.f., [1], pp. $7 \sim 8$ ).

DEFINITION 5.5. Let $\varphi(\not \equiv 0)$ be a meromorphic function on $B(R)$. Define

$$
m(r, \varphi):=\frac{1}{\Phi(r)} \int_{S(r)} \log ^{+}|\varphi(z)| \sigma_{r}(z)
$$

where $\log ^{+} x=\max (\log x, 0)$ for any $x \geqq 0$.

(5.6) If $0 \notin\left|\nu_{\varphi}^{0}\right| \cup\left|\nu_{\varphi}^{\infty}\right|$ for a meromorphic function $\varphi(z)$ on $B(R)$, then

$$
T(r, \varphi)=T\left(r, \frac{1}{\varphi}\right)=N\left(r, \nu_{\varphi}^{\infty}\right)+m(r, \varphi)-\log ^{+}|\varphi(0)| .
$$

This is an immediate consequence of Jensen's formula (2.4) as in [1], p. 9.

DEFINITION 5.7. Let $f=f_{0}: f_{1}: \cdots: f_{N}$ be an admissible representation of a meromorphic map $f$ of $B(R)$ into $P_{N}(C)$. Take a hyperplane

$$
H: a^{0} w_{0}+a^{1} w_{1}+\cdots+a^{N} w_{N}=0
$$

with $f(0) \notin H$, where we assume $\sum_{i=0}^{N}\left|a^{i}\right|^{2}=1$. Putting $F:=a^{0} f_{0}+a^{1} f_{1}$ $+\cdots+a^{N} f_{N}$, we define

$$
\Psi(f, H):=\log |F(0)|-\max _{0 \leqq i \leqq N} \log \left|f_{i}(0)\right| .
$$

Take another hyperplane

$$
H^{\prime}: b^{0} w_{0}+b^{1} w_{1}+\cdots+b^{N} w_{N}=0
$$

with $f(B(R)) \not \subset H^{\prime}$ and put $F^{\prime}:=b^{0} f_{0}+\cdots+b^{N} f_{N}$. We define

$$
\varphi\left(f ; H, H^{\prime}\right):=\frac{F}{F^{\prime}} .
$$

(5.8) Suppose that $f(0) \notin H \cup H^{\prime}$. Then,

$$
T\left(r, \varphi\left(f ; H, H^{\prime}\right)\right) \leqq T(r, f)-\Psi\left(f, H^{\prime}\right)+K
$$


where $K$ is a constant depending only on $H$ and $H^{\prime}$.

This will be shown by giving the precise estimation of constant terms of inequalities obtained in [1], p. 10. By (5.4) it may be assumed that $H=\left\{w_{0}=0\right\}$ and $H^{\prime}=\left\{w_{1}=0\right\}$. We can write $\varphi:=\varphi\left(f ; H, H^{\prime}\right)=g_{0} / g_{1}$ with holomorphic functions $g_{i}$ such that $\operatorname{codim}\left\{g_{0}=g_{1}=0\right\} \geqq 2$ and $u_{1}(0)=0$ for $u_{1}(z):=\max _{i=0,1} \log \left|g_{i}(z)\right|$. Then, putting $h=f_{0} / g_{0}\left(=f_{1} / g_{1}\right)$, we have

$$
\begin{aligned}
T(r, f) & \geqq \frac{1}{\Phi(r)} \int_{S(r)}\left(u_{1}(z)+\log |h(z)|\right) \sigma_{r}(z)-u(0) \\
& \geqq T(r, \varphi)+u_{1}(0)+\log |h(0)|+N\left(r, \nu_{h}\right)-u(0) \\
& \geqq T(r, \varphi)+\log |h(0)|-u(0)
\end{aligned}
$$

On the other hand,

$$
\log |h(0)|=\log \left|f_{1}(0)\right|-\log \left|g_{1}(0)\right| \geqq \log \left|f_{1}(0)\right|
$$

because $\log \left|g_{1}(0)\right| \leqq u_{1}(0)=0$. This leads to (5.8).

(5.9) Let $H$ be a hyperplane with $f(0) \notin H$. Then,

$$
N(r, \nu(f, H)) \leqq T(r, f)-\Psi(f, H)+K,
$$

where $K$ is a constant depending only on $H$.

To see this, refer to [1], p. 11.

As is easily seen, a family $\mathscr{F}$ of holomorphic functions on the unit disc is normal if $m(r, f)(f \in \mathscr{F})$ are uniformly bounded. We shall generalize this to the case of meromorphic maps into $P_{N}(C)$.

THEOREM 5.10. Let $\mathscr{F}$ be a family of meromorphic maps of $B(R)$ into $P_{N}(C)$ each of which is holomorphic at the origin. The characteristic functions $T(r, f)(f \in \mathscr{F}, 0<r<R)$ are bounded by a constant depending only on $r$ if and only if $\mathscr{F}$ is m-normal and normal at the origin.

Proof. Suppose that

$$
T(r, f) \leqq K_{r} \quad(f \in \mathscr{F})
$$

for a constant $K_{r}(0<r<R)$. Take an arbitrary sequence $\left\{f^{(p)}\right\}$ in $\mathscr{F}$. Replacing it by a suitable subsequence, we may assume that $\lim _{p \rightarrow \infty} f^{(p)}(0)$ $=v$ exists in $P_{N}(C)$. Choose $2 N+1$ hyperplanes $H_{j}(0 \leqq j \leqq 2 N)$ in general position such that $v \notin \bigcup_{j=0}^{2 N} H_{j}$ and consider the quantities $\Psi\left(f^{(p)}, H_{j}\right)$ 
as in Definition 5.7 for arbitrarily fixed homogeneous coordinates. Then, $\left\{\Psi\left(f^{(p)}, H_{j}\right): p=1,2, \cdots\right\}$ are all convergent and so we can find a constant $K^{\prime}(>0)$ such that

$$
-\Psi\left(f^{(p)}, H_{j}\right) \leqq K^{\prime}
$$

for any $p$ and $j$. Therefore, by (5.9)

$$
N\left(r, \nu\left(f^{(p)}, H_{j}\right)\right) \leqq K_{r}+K^{\prime}+K,
$$

where $K$ is a constant not depending on each $p$. Now apply Corollary 4.5 to the family $\left\{f^{(p)}\right\}$. We can choose a subsequence $\left\{f^{\left(p_{k}\right)}\right\}$ of $\left\{f^{(p)}\right\}$ which is $m$-convergent on $B(R)$. To show that $f$ is normal at the origin, we have only to prove that there exists a neighborhood $U$ of the origin, such that $U \cap\left|\nu\left(f^{\left(p_{k}\right)}, H_{0}\right)\right|=\phi$ for all but finitely many $k$. In fact, in this case, choosing homogeneous coordinates $w_{0}: w_{1}: \cdots: w_{N}$ with $H_{0}=$ $\left\{w_{0}=0\right\}$, we can take admissible representations

$$
f^{\left(p_{k}\right)}=1: \varphi_{1}^{\left(p_{k)}\right)}: \varphi_{2}^{\left(p_{k)}\right)}: \cdots: \varphi_{N}^{\left(p_{k}\right)}
$$

with holomorphic functions $\varphi_{i}^{\left(p_{k}\right)}$ on $U$ such that $\lim _{k \rightarrow \infty} \varphi_{i}^{\left(p_{k}\right)}$ exist, which leads to the desired conclusion. Assume the contrary, i.e., for any $\delta>0$ let infinitely many $p_{k}$ satisfy the condition

$$
B(\delta) \cap\left|\nu\left(f^{\left(p_{k}\right)}, H_{0}\right)\right| \neq \phi .
$$

Then, for such $p_{k}$, by Lemma 1.10 in [16]

$$
V\left(t, \nu\left(f^{\left(p_{k}\right)}, H\right)\right) \geqq W(t-\delta)^{2 n-2},
$$

where $\delta \leqq t<R$. Therefore, we have

$$
N\left(r, \nu\left(f^{\left(p_{k}\right)}, H_{0}\right)\right) \geqq \int_{\delta}^{r} \frac{V\left(t, \nu\left(f^{\left(p_{k}\right)}, H_{0}\right)\right)}{W t^{2 n-1}} d t \geqq \int_{\delta}^{r} \frac{(t-\delta)^{2 n-2}}{t^{2 n-1}} d t .
$$

On the other hand,

$$
\lim _{\delta \rightarrow 0} \int_{\delta}^{r} \frac{(t-\delta)^{2 n-2}}{t^{2 n-1}} d t=\infty
$$

This shows that, for a fixed $r, N\left(r, \nu\left(f^{\left(p_{k}\right)}, H_{0}\right)\right)$ becomes larger than any given number if a sufficiently large $k$ is taken, which is a contradiction.

Conversely, suppose that $\mathscr{F}$ is $m$-normal and normal at the origin and, moreover, there exists some $r(0<r<R)$ such that, for each $p$, at least one $f \in \mathscr{F}$, say $f^{(p)}$, satisfies the condition 


$$
T\left(r, f^{(p)}\right) \geqq p
$$

After a suitable change of indices, it may be assumed that $\left\{f^{(p)}\right\}$ converges meromorphically on $B(R)$ to a meromorphic map $f$ and converges compactly on $B(\delta)$ for some $\delta>0$. In this situation, using Proposition 3.8 we can choose admissible representations

$$
f^{(p)}=f_{0}^{(p)}: f_{\mathrm{i}}^{(p)}: \cdots: f_{N}^{(p)}
$$

on $B\left(r^{\prime}\right)$ such that $\lim _{p \rightarrow \infty} f_{i}^{(p)}=f_{i}$ exists on $B\left(r^{\prime}\right)$ and $f=f_{0}: f_{1}: \cdots: f_{N}$ and, moreover, $\left\{f_{0}=f_{1}=\cdots=f_{N}=0\right\} \cap B(\delta)=\phi$, where $r^{\prime}\left(r<r^{\prime}<R\right)$ is arbitrarily fixed. Put

$$
u_{p}(z):=\max _{0 \leqq i \leqq N} \log \left|f_{i}^{(p)}(z)\right|
$$

and

$$
u(z):=\max _{0 \leqq i \leqq N} \log \left|f_{i}(z)\right| .
$$

Obviously, $\left\{u_{p}(z)\right\}$ converges compactly to $u(z)$ on $B\left(r^{\prime}\right)-\{u(z)=-\infty\}$ and, particularly, on the totality of $B(\delta)$. There is a positive constant $M$ such that

$$
u_{p}(0) \geqq-M
$$

for any $p$. On the other hand, since $S:=\{u(z)=-\infty\}$ is a thin analytic subset of $B\left(r^{\prime}\right)$, we can find a compact set $C$ in $B\left(r^{\prime}\right)-S$ such that

$$
\sup \{v(z): z \in \overline{B(r)}\} \leqq \sup \{v(z): z \in C\}
$$

for any plurisubharmonic function $v(z)$ on $B\left(r^{\prime}\right)$. This concludes that $\left\{u_{p}(z) ; z \in B(r), p=1,2, \cdots\right\}$ are bounded above by a constant $M^{\prime}$ uniformly. In conclusion, we have

$$
\begin{aligned}
T\left(r, f^{(p)}\right) & =\frac{1}{\Phi(r)} \int_{S(r)} u_{p}(z) \sigma_{r}(z)-u_{p}(0) \\
& \leqq M^{\prime}+M
\end{aligned}
$$

which is a contradiction. The proof of Theorem 5.10 is complete.

\section{§6. The fundamental inequality.}

Let $H_{j}(1 \leqq j \leqq q)$ be $q(\geqq N+2)$ hyperplanes in general position and $f$ a meromorphic map of $B(R)$ into $P_{N}(C)$ satisfying the condition 
$f(0) \notin \bigcup_{j=1}^{q} H_{j}$. We may take homogeneous coordinates $w_{0}: w_{1}: \cdots: w_{N}$ on $P_{N}(C)$ such that $H_{i+1}=\left\{w_{i}=0\right\}(0 \leqq i \leqq N)$. Let $f=f_{0}: f_{1}: \cdots: f_{N}$ be an admissible representation $f$ on $B(R)$, where $f_{i}(0) \neq 0(0 \leqq i \leqq N)$. We expand each $f_{i}$ as

$$
f_{i}(z)=\sum_{m=0}^{\infty} P_{i}^{m}(z)
$$

on $B(R)$, where $P_{i}^{m}(z)$ is either identically zero or homogeneous polynomial of degree $m$. Consider the function

$$
W_{f}(z):=\frac{1}{P_{0}^{0} P_{1}^{0} \cdots P_{N}^{0}}\left|\begin{array}{c}
P_{0}^{0}, P_{1}^{0}, \cdots, P_{N}^{0} \\
P_{0}^{1}, P_{1}^{1}, \cdots, P_{N}^{1} \\
\cdots \cdots \cdots \\
P_{0}^{N}, P_{1}^{N}, \cdots, P_{N}^{N}
\end{array}\right|
$$

which is a homogeneous polynomial of degree $N(N+1) / 2$ and uniquely determined independently of a choice of admissible representations.

DEFINITION 6.1. We define

$$
W_{f}^{*}:=\frac{1}{\Phi(1)} \int_{S(1)} \log \left|W_{f}(z)\right| \sigma_{1}(z)
$$

Remark. (i) The quantity $W_{f}^{*}$ is determined only by the values of $\leqq N$-th derived functions of $f_{i}$ at the origin. For the particular case $n=1, W_{f}(1)$ is nothing but the values of the Wronskian of a system of holomorphic functions $f_{0}, f_{1}, \cdots, f_{N}$ at the origin divided by a constant multiple of $f_{0}(0) f_{1}(0) \cdots f_{N}(0)$.

(ii) Put $f_{a}^{\sharp}(u)=f(a u)$ for almost all $a \in S(1)$. As is easily seen,

$$
W_{f}^{*}=\frac{1}{\Phi(1)} \int_{a \in S(1)} W_{f_{a}^{\#}}^{*} \sigma_{1}(a)
$$

Now, we give the following fundamental inequality.

THEOREM 6.2. In the above situation, it holds that, for any $r$ $(0<r<R)$,

$$
(q-N-1) T(r, f) \leqq \sum_{j=1}^{q} N_{N}\left(r, \nu\left(f, H_{j}\right)\right)+S(r),
$$

where, for any given $\varepsilon(>0)$ and $\rho(r<\rho<R)$, $S(r)$ is evaluated as follows; 


$$
\begin{aligned}
S(r) \leqq & K_{0}+K_{1} \log ^{+} \rho+K_{2} \log ^{+} \frac{1}{\rho-r}+K_{3} \log ^{+} \frac{1}{r} \\
& +K_{4} \sum_{j=1}^{q} \log ^{+}\left|\Psi\left(f, H_{j}\right)\right|+\sum_{j=N+2}^{q} \Psi\left(f, H_{j}\right) \\
& +\varepsilon T(\rho, f)-W_{f}^{*}
\end{aligned}
$$

with constants $K_{0}$ depending only on $\varepsilon, H_{j}(1 \leqq j \leqq q)$ and with constants $K_{\kappa}(1 \leqq \kappa \leqq 4)$ depending only on $N$.

We consider first Theorem 6.2 for the case $n=1$. In [1], it was proved that the quantity $S(r)$ in Theorem 6.2 is evaluated as

$$
S(r) \leqq L_{1} \sum_{j=2}^{q} \sum_{\ell=2}^{N+1} m\left(r, \frac{d^{\ell-1}}{d z^{\ell-1}}\left(\frac{\varphi_{j}^{\prime}}{\varphi_{j}}\right)\right)+L_{2}
$$

with some constants $L_{1}$ and $L_{2}$, where $\varphi_{j}:=\varphi\left(f ; H_{j}, H_{1}\right)$ (c.f. Definition 5.7) and, as its consequence,

$$
S(r)=O\left(\log ^{+} T(r, f)\right)+O\left(\log ^{+} \frac{1}{R-r}\right)
$$

outside some sufficiently small set of $r$ in the case $n=1$ and $R<\infty$. For the proof of Theorem 6.2, we have to obtain more precise estimation of $S(r)$. By observing H. Cartan's proof of (6.3) ([1], pp. 12-15) carefully, it is not difficult to ascertain that the constant $L_{1}$ of (6.3) can be chosen independently of each $r, f$ and, moreover, the constant $L_{2}$ can be replaced by

$$
M+\sum_{j=N+2}^{q} \Psi\left(f, H_{j}\right)-W_{f}^{*},
$$

where $M$ is a constant depending only on $H_{j}(1 \leqq j \leqq q)$. To estimate the other terms, we need

LEMMA 6.5. Let $\varphi(z)$ be a meromorphic function on $\{|z|<R\}(\subset C)$ with $\varphi(0) \neq 0, \infty$. For any $r, \rho(0<r<\rho<R)$ and a positive integer $\ell$, there are some constants $K_{\kappa}^{\prime}(0 \leqq \kappa \leqq 5)$ depending only on $\ell$ such that

$$
\begin{aligned}
m\left(r, \frac{d^{\ell-1}}{d z^{\ell-1}}\left(\frac{\varphi^{\prime}(z)}{\varphi(z)}\right)\right) \leqq & K_{0}^{\prime}+K_{1}^{\prime} \log ^{+} \rho+K_{2}^{\prime} \log ^{+} \frac{1}{\rho-r}+K_{3}^{\prime} \log ^{+} \frac{1}{r} \\
& +K_{4}^{\prime} \log ^{+}|\log | \varphi(0)||+K_{5}^{\prime} \log ^{+} T(\rho, \varphi) .
\end{aligned}
$$

Proof. We can prove Lemma 6.5 along the same lines of the proof of Lemma 2.3 in [8] by adding some considerations. We describe here only the outline of the proof. At first, suppose that $\varphi(z) \neq 0, \infty$ on $\{|z|=r\}$ 
$\bigcup\{|z|=\rho\}$. We denote by $a_{\mu}\left(1 \leqq \mu \leqq n_{0}\right)$ all zeros of $\varphi(z)$ in $\{|z| \leqq \rho\}$ and by $b_{\nu}\left(1 \leqq \nu \leqq n_{\infty}\right)$ all poles of $\varphi(z)$ in $\{|z| \leqq \rho\}$. By differentiating the well-known Poisson-Jensen's formula (e.g., [8], p. 1) $\ell$ times, we have

$$
\begin{aligned}
\frac{d^{\ell-1}}{d z^{\ell-1}}\left(\frac{\varphi^{\prime}(z)}{\varphi(z)}\right)= & \frac{\ell !}{2 \pi} \int_{0}^{2 \pi} \log \left|\varphi\left(\rho e^{i \theta}\right)\right| \frac{2 \rho e^{i \theta}}{\left(\rho e^{i \theta}-z\right)^{l+1}} d \theta \\
& -(\ell-1) ! \sum_{\mu=1}^{n_{0}}\left(\frac{1}{\left(a_{\mu}-z\right)^{\ell}}-\frac{\bar{a}_{\mu}^{\ell}}{\left(\rho^{2}-\bar{a}_{\mu} z\right)^{\ell}}\right) \\
& +(\ell-1) ! \sum_{\nu=1}^{n_{\infty}}\left(\frac{1}{\left(b_{\nu}-z\right)^{\ell}}-\frac{\bar{b}_{\nu}^{\ell}}{\left(\rho^{2}-\bar{b}_{\nu} z\right)^{\ell}}\right),
\end{aligned}
$$

where $0<r=|z|<\rho$. Put $\delta(z):=\min _{\mu, \nu}\left\{\left|z-a_{\mu}\right|,\left|z-b_{\nu}\right|\right\}$ and $n(\rho):=$ $n_{0}+n_{\infty}$. As in the proof of Lemma 2.3 in [8], by the inequalities

$$
\left|\frac{\bar{a}_{\mu}}{\rho^{2}-\bar{a}_{\mu} z}\right|^{\ell} \leqq\left(\frac{1}{\rho-r}\right)^{\ell}, \quad \frac{1}{\left|a_{\mu}-z\right|^{\ell}} \leqq \frac{1}{\delta(z)^{\ell}}
$$

and

$$
\begin{aligned}
\frac{1}{2 \pi} \int_{0}^{2 \pi}|\log | \varphi\left(r e^{i \theta}\right) \| d \theta & =m(r, \varphi)+m\left(r, \frac{1}{\varphi}\right) \\
& \leqq 2 T(r, \varphi)+|\log | \varphi(0)||
\end{aligned}
$$

etc., we get

$$
\begin{aligned}
\left|\frac{d^{\ell-1}}{d z^{\ell-1}}\left(\frac{\varphi^{\prime}(z)}{\varphi(z)}\right)\right| \leqq & \frac{2 \ell ! \rho}{(\rho-r)^{\ell+1}}\{2 T(\rho, \varphi)+|\log | \varphi(0)||\} \\
& +\frac{n(\rho)(\ell-1) !}{r^{\ell}}\left\{\left(\frac{r}{\delta(z)}\right)^{\ell}+\left(\frac{r}{\rho-r}\right)^{\ell}\right\} .
\end{aligned}
$$

Since $\log ^{+} x_{1} x_{2} \cdots x_{n} \leqq \sum_{i=1}^{n} \log ^{+} x_{i}$ and $\log ^{+}\left(\sum_{i=1}^{n} x_{i}\right) \leqq \log n+\sum_{i=1}^{n} \log ^{+} x_{i}$ for any $x_{i} \geqq 0$, we obtain by the use of Lemma 2.2 in [8]

$$
\begin{aligned}
m(r, & \left.\frac{d^{\ell-1}}{d z^{\ell-1}}\left(\frac{\varphi^{\prime}(x)}{\varphi(z)}\right)\right) \equiv \frac{1}{2 \pi} \int_{0}^{2 \pi} \log ^{+}\left|\frac{d^{\ell-1}}{d z^{\ell-1}}\left(\frac{\varphi^{\prime}}{\varphi}\right)\left(r e^{i \theta}\right)\right| d \theta \\
\leqq & K^{*}+\log ^{+} \rho+\ell \log ^{+} r+(2 \ell+1) \log ^{+} \frac{1}{\rho-r}+\ell \log ^{+} \frac{1}{r} \\
& +\log ^{+}|\log | \varphi(0)||+\log ^{+} T(\rho, \varphi)+(2 \ell+1) \log ^{+} n(\rho) .
\end{aligned}
$$

for an absolute constant $K^{*}$. We note here that (6.6) remains valid without the assumption $\varphi(z) \neq 0, \infty$ on $\{|z|=r\} \cup\{|z|=\rho\}$, because the both sides of (6.6) are continuous functions of $r$ and upper semi-continuous functions of $\rho$. 
Now, for arbitrarily given $r$ and $\rho(0<r<\rho<R)$, replace $\rho$ in (6.6) by $\rho^{\prime}:=(\rho+r) / 2$. As in [8], p. 37,

$$
n\left(\rho^{\prime}\right) \leqq \frac{\rho}{\rho-\rho^{\prime}}\{2 T(\rho, \varphi)+|\log | \varphi(0)||\}
$$

and hence

$$
\begin{aligned}
\log ^{+} n(\rho) \leqq & \log ^{+} \rho+\log ^{+} \frac{2}{\rho-r}+\log ^{+} T(\rho, \varphi) \\
& +\log ^{+}|\log | \varphi(0)||+K
\end{aligned}
$$

for some constant $K$. By substituting this into (6.6) and using the monotone property of functions $\log ^{+} \rho$ and $T(\rho, \varphi)$, we can easily obtain the desired conclusion.

Proof of Theorem 6.2. By (6.3), (6.4) and Lemma 6.5, we can write

$$
\begin{aligned}
S(r) \leqq & K_{0}^{\prime \prime}+K_{1}^{\prime \prime} \log ^{+} \rho+K_{2}^{\prime \prime} \log ^{+} \frac{1}{\rho-r}+K_{3}^{\prime \prime} \log ^{+} \frac{1}{r} \\
& +K_{4}^{\prime \prime} \sum_{j=2}^{q} \log ^{+}|\log | \varphi_{j}(0)||+\sum_{j=N+2}^{q} \Psi\left(f, H_{j}\right) \\
& +K_{5}^{\prime \prime} \sum_{j=2}^{q} \log ^{+} T\left(\rho, \varphi_{j}\right)-W_{f}^{*}
\end{aligned}
$$

with some constants $K_{\kappa}^{\prime \prime}(0 \leqq \kappa \leqq 5)$. On the other hand,

$$
\begin{aligned}
\log ^{+}|\log | \varphi_{j}(0)|| & \leqq \log ^{+}\left|\Psi\left(f, H_{j}\right)-\Psi\left(f, H_{1}\right)\right| \\
& \leqq \log ^{+}\left|\Psi\left(f, H_{j}\right)\right|+\log ^{+}\left|\Psi\left(f, H_{1}\right)\right|+\log 2
\end{aligned}
$$

by Definition 5.7 and

$$
\log ^{+} T\left(\rho, \varphi_{j}\right) \leqq \log ^{+} T(\rho, f)+\log ^{+}\left|\Psi\left(f, H_{1}\right)\right|+K
$$

for some constant $K$ by (5.8). We can rewrite (6.7) as

$$
\begin{aligned}
S(r) \leqq & K_{0}+K_{1} \log ^{+} \rho+K_{2} \log ^{+} \frac{1}{\rho-r}+K_{3} \log ^{+} \frac{1}{r} \\
& +K_{4} \sum_{j=1}^{q} \log ^{+}\left|\Psi\left(f, H_{j}\right)\right|+\sum_{j=N+2}^{q} \Psi\left(f, H_{j}\right) \\
& +K_{5} \log ^{+} T(\rho, f)-W_{f}^{*}
\end{aligned}
$$

with suitable constants $K_{\kappa}(0 \leqq \kappa \leqq 5)$. Then, replacing the above $K_{0}$ by a new constant, maybe depending on $\varepsilon$, we can conclude the desired fundamental inequality, because there is a positive constant $K(\varepsilon)$ such that $K_{4} \log ^{+} x \leqq \varepsilon x+K(\varepsilon)$ for any $x \geqq 0$.

Now, we proceed to the proof of Theorem 6.2 for the general case. 
Consider the meromorphic map $(f)_{z}^{\sharp}(u):=f(z u)$ for any $z \in S(1)$ except a set of measure zero. Using the above shown result for the case $n=$ 1 , we have

$$
\begin{aligned}
(q- & N-1) T\left(r, f_{z}^{\sharp}\right) \leqq \sum_{j=1}^{q} N_{N}\left(r, \nu\left(f_{z}^{\sharp}, H_{j}\right)\right)+K_{0} \\
& +K_{1} \log ^{+} \rho+K_{2} \log ^{+} \frac{1}{\rho-r}+K_{3} \log ^{+} \frac{1}{r} \\
& +K_{4} \sum_{j=1}^{q} \log ^{+}\left|\Psi\left(f, H_{j}\right)\right|+\sum_{j=N+2}^{q} \Psi\left(f, H_{j}\right) \\
& +\varepsilon T\left(\rho, f_{z}^{\sharp}\right)-W_{f_{z}^{*}}^{*}
\end{aligned}
$$

for any $r$, $\rho$ with $0<r<\rho<R$. We calculate the mean values of $S(1)$ of both sides of (6.8). By (5.3), (2.3) and Remark (ii) to Definition 6.1, we conclude Theorem 6.2.

Remark. In Theorem 6.2, if $R=\infty$ and $f$ is rational, i.e., $f$ has a representation $f=f_{0}: f_{1}: \cdots: f_{N}$ with polynomials $f_{i}$, then we can show easily $S(r)=O(1)$.

\section{\$7. A defect relation for meromorphic maps into $P_{N}(C)$.}

Let $f$ be a meromorphic map of $B(R)$ into $P_{N}(C)$ which is assumed to be holomorphic at the origin and $H$ a hyperplane with $f(B(R)) \not \subset H$.

DEFINITION 7.1. We define the modified deficient function as

$$
\delta_{m}(f, H):=1-\varlimsup_{r \rightarrow R} \frac{N_{m}(r, \nu(f, H))}{T(r, f)},
$$

where $0<m \leqq \infty$. And, we put $\delta(f, H):=\delta_{\infty}(f, H)$.

As is easily seen, we have $0 \leqq \delta(f, H) \leqq 1$, if $\lim _{r \rightarrow R} T(r, f)=\infty$.

DEFINITION 7.2. We shall call $f$ to be transcendental if and only if

$$
\lim _{r \rightarrow \infty} \frac{T(r, f)}{\log r}=\infty \quad \text { in case of } R=\infty
$$

and

$$
\lim _{r \rightarrow \infty} \frac{T(r, f)}{\log (1 /(R-r))}=\infty \quad \text { in case of } R<\infty
$$

(7.3) For a meromorphic map $f$ of $C^{n}$ into $P_{N}(C), f$ is not transcendental if and only if $f$ is rational. 
This is a result of W. Stoll ([14], Satz 24.1). Because, according to Remark (ii) to Definition 5.1 the transcendency of $f$ is equal to what was defined by W. Stoll in [14].

Now, we give a defect relation for a meromorphic map.

THEOREM 7.4. Let $f$ satisfy the condition $W_{f}^{*} \neq-\infty$ and $H_{j}(1 \leqq j \leqq q)$ be arbitrarily given hyperplanes in the $P_{N}(C)$ located in general position such that $f(0) \notin \bigcup_{j=0}^{q} H_{j}$. If $R=\infty$ or $R<\infty$ and $f$ is transcendental, then

$$
\sum_{j=1}^{q} \delta_{N}\left(f, H_{j}\right) \leqq N+1 .
$$

Remark. Since $\delta\left(f, H_{j}\right) \leqq \delta_{N}\left(f, H_{j}\right)$, we have

$$
\sum_{j=1}^{q} \delta\left(f, H_{j}\right) \leqq N+1
$$

as a consequence of Theorem 7.4. This is a special case of a defect relation given by W. Stoll in [14], §23.

Proof of Theorem 7.4. We shall prove Theorem 7.4 in the case $R<\infty$ only, because the analogous argument can be applied for the case $R=\infty$ too with some simple modifications. Since $\lim _{r \rightarrow R} T(r, f)=\infty$ by the assumption and $T(r, f)$ is a monotone increasing continuous function of $r$, we have by Lemma 2.4 in [8]

$$
T\left(r+\frac{R-r}{e T(r, f)}, f\right) \leqq 2 T(r, f)
$$

outside a set $E_{0}$ of $r$ with

$$
\int_{E_{0}} \frac{d r}{R-r}<\infty
$$

Therefore, we can choose some $r \notin E_{0}(0<r<R)$ which is arbitrarily close to $R$. Substitute $\rho=r+[(R-r) / e T(r, f)]$ into the fundamental inequality in Theorem 6.2. Then, for any $\varepsilon>0$,

$$
\begin{aligned}
S(r) \leqq & L_{0}+L_{1} \log ^{+}\left(r+\frac{R-r}{e T(r, f)}\right)+L_{2} \log ^{+} \frac{e T(r, f)}{R-r} \\
& +L_{3} \log ^{+} \frac{1}{r}+2 \varepsilon T(r, f)
\end{aligned}
$$

by the assumption $W_{f}^{*} \neq-\infty$, where $S(r)$ is the quantity given in Theorem 6.2 and $L_{\kappa}(0 \leqq \kappa \leqq 3)$ are constants not depending on each $r$. 
It can be rewritten

$$
S(r) \leqq L_{0}^{\prime}+L_{1}^{\prime} \log ^{+} T(r, f)+L_{2}^{\prime} \log \frac{1}{R-r}+2 \varepsilon T(r, f)
$$

with new constants $L_{x}^{\prime}(0 \leqq \kappa \leqq 2)$ if $r$ is sufficiently close to $R$. Thus, we have

$$
\varliminf_{r \rightarrow R} \frac{S(r)}{T(r, f)} \leqq 2 \varepsilon
$$

because $f$ is transcendental. Consequently,

$$
\begin{aligned}
\sum_{j=1}^{q} \delta\left(f, H_{j}\right) & =\varliminf_{r \rightarrow R}\left(q-\sum_{j=1}^{q} \frac{N_{N}\left(r, \nu\left(f, H_{j}\right)\right)}{T(r, f)}\right) \\
& \leqq N+1+\varliminf_{r \rightarrow R} \frac{S(r)}{T(r, f)} \leqq N+1+2 \varepsilon .
\end{aligned}
$$

Since $\varepsilon$ is arbitrary, we conclude the desired defect relation.

We examine here the meaning of the condition $W_{f}^{*}=-\infty$ in the case $R=\infty$. Consider first the case $n=1$. In this case, $W_{f}^{*}=-\infty$ means that the Wronskian $W\left(f_{0}, f_{1}, \cdots, f_{N}\right)$ of a system of holomorphic functions $f_{0}, f_{1}, \cdots, f_{N}$ vanishes at the origin for an admissible representation $f=f_{0}: f_{1}: \cdots: f_{N}$ on $C^{n}$. If $W\left(f_{0}, f_{1}, \cdots, f_{N}\right) \not \equiv 0$, namely, the image of $f$ are not included in any hyperplane in $P_{N}(C)$, then we can choose global coordinates on $C^{n}$ such that $f$ satisfies all assumptions of Theorem 7.4. Let us consider next the case $n \geqq 2$. Since $\log \left|W_{f}(z)\right|$ is a plurisubharmonic function of $z$,

$$
M(r):=\frac{1}{\Phi(r)} \int_{z \in S(r)} \log \left|W_{f}(z)\right| \sigma_{r}(z)
$$

is a monotone increasing function of $r$. Therefore, $W_{f}^{*}=M(1)=-\infty$ implies that $M(r)=-\infty$ for any $r>0$ and hence $W_{f}(z) \equiv 0$. And, this holds if and only if $W\left(\left(f_{0}\right)_{z}^{\#},\left(f_{1}\right)_{z}^{\#}, \cdots,\left(f_{N}\right)_{z}^{\#}\right)$ vanishes at the origin for any $z \in S(1)$, where we put $\left(f_{i}\right)_{z}^{*}(u):=f_{i}(z u)$ for an admissible representation $f=f_{0}: f_{1}: \cdots: f_{N}$. If there exists at least one complex line $\ell$ such that $\ell \not \subset f^{-1}(H)$ for any hyperplane $H$ in $P_{N}(C)$, then the assumptions of Theorem 7.4 are all satisfied after a suitable change of global coordinates on $C^{n}$. In the particular case $N=1$, this means that $f$ is not of constant. 
COROLLARY 7.5 (c.f., [1], p. 19). Let $f$ be a meromorphic map of $C^{n}$ into $P_{N}(C)$ such that, for some complex line $\ell$ in $C^{n}, f$ is holomorphic at a point in $\ell$ and $f(\ell)$ cannot be included in any hyperplane in $P_{N}(C)$. Suppose that there exist $q(\geqq N+2)$ hyperplanes $H_{j}(1 \leqq j \leqq q)$ in general position such that, for some positive integers $m_{j}(1 \leqq j \leqq q), \nu\left(f, H_{j}\right) \geqq m_{j}$ everywhere on $\left|\nu\left(f, H_{j}\right)\right|$ for any $j$. Then

$$
\sum_{j=1}^{q} \frac{1}{m_{j}} \geqq \frac{q-N-1}{N}
$$

Proof. As is stated above, after a suitable change of global coordidates we may assume that $f(0) \notin \bigcup_{j=1}^{q} H_{j}$ and $W_{f}^{*} \neq-\infty$. We have

$$
\begin{aligned}
N_{N}\left(r, \nu\left(f, H_{j}\right)\right) & \leqq N \cdot N_{1}\left(r, \nu\left(f, H_{j}\right)\right) \leqq \frac{N}{m_{j}} N\left(r, \nu\left(f, H_{j}\right)\right) \\
& \leqq \frac{N}{m_{j}}(T(r, f)+M)
\end{aligned}
$$

by (5.9) for some constant $M$ not depending on $r$ and so

$$
\delta_{N}\left(f, H_{j}\right) \geqq 1-\frac{N}{m_{j}} .
$$

This gives Corollary 7.5 as a consequence of Theorem 7.4.

We can give also another application of Theorem 7.4.

COROLlaRY 7.6. For a meromorphic map $f$ with the same property as in Corollary 7.5, suppose that there exist $N+2$ hyperplanes $H_{j}(1 \leqq j$ $\leqq N+2)$ in general position such that each $f^{-1}\left(H_{j}\right)$ is an algebraic set in $\boldsymbol{C}^{n}$. Then $f$ is necessarily rational.

Proof. Assume that $f$ is transcendental. As is well-known, for a divisor $\nu$ on $C^{n}|\nu|$ is algebraic if and only if there exists some constant $K$ not depending on each $r$ such that

$$
\frac{V_{1}(r, \nu)}{r^{2 n-2}} \leqq K
$$

(c.f., [13], Satz 23, for the case $n=2$ and [15], Theorem 7.15, for the general case). We can easily conclude from the assumption that

$$
\lim _{r \rightarrow \infty} \frac{N_{1}\left(r, \nu\left(f, H_{j}\right)\right)}{\log r}<\infty \quad(1 \leqq j \leqq N+2)
$$


Therefore,

$$
\varlimsup_{r \rightarrow \infty} \frac{N_{N}\left(r, \nu\left(f, H_{j}\right)\right)}{T(r, f)} \leqq N \varlimsup_{r \rightarrow \infty} \frac{N_{1}\left(r, \nu\left(f, H_{j}\right)\right)}{\log r} \frac{\log r}{T(r, f)}=0,
$$

which means $\delta\left(f, H_{j}\right)=1$ for any $j(1 \leqq j \leqq N+2)$. We have thus a contradiction

$$
N+1 \geqq \sum_{j=1}^{N+2} \delta\left(f, H_{j}\right)=N+2 .
$$

This completes the proof.

Remark. Theorem 7.6 was firstly shown by $P$. Thullen ([17]) for the case $N=1$.

\section{§ 8. A sufficient condition for $m$-normal families.}

Let $\mathscr{F}$ be a family of meromorphic maps of $B(R)$ into $P_{N}(C)$ each of which is assumed to be holomorphic at the origin. As a consequence of Theorem 6.2, we can give the following sufficient condition for $\mathscr{F}$ to be $m$-normal.

THEOREM 8.1. Suppose that there exist $q(\geqq N+2)$ hyperplanes $H_{j}(1 \leqq j \leqq q)$ in $P_{N}(C)$ satisfying the condition that

(i) the values $f(0)(f \in \mathscr{F})$ are contained in a fixed compact subset of $P_{N}(C)-\bigcup_{j=1}^{q} H_{j}$,

(ii) $W_{f}^{*}(f \in \mathscr{F})$ are bounded below by a fixed constant and

(iii) $\nu\left(f, H_{j}\right) \geqq m_{j}$ everywhere on $\left|\nu\left(f, H_{j}\right)\right|$ for some fixed positive integers $m_{j}(1 \leqq j \leqq q)$ with

$$
\sum_{j=1}^{q} \frac{1}{m_{j}}<\frac{q-N-1}{N} .
$$

Then, $\mathscr{F}$ is m-normal and normal at the origin.

Proof. As in the proof of Corollary 7.5,

$$
N_{N}\left(r, \nu\left(f, H_{j}\right)\right) \leqq \frac{N}{m_{j}} T(r, f)+K_{j}
$$

for some constant $K_{j}(1 \leqq j \leqq q)$ because $\left|\Psi\left(f, H_{j}\right)\right|(f \in \mathscr{F})$ are uniformly bounded by the assumption (i), where $0<r<R$. Therefore, we have

$$
\left((q-N-1)-\sum_{j=1}^{q} \frac{N}{m_{j}}\right) T(r, f) \leqq S(r)+\Sigma_{j} K_{j}
$$


where $S(r)$ is the quantity given in Theorem 6.2. Put $\delta:=q-N-1-$ $\sum_{j=1}^{q}\left(N / m_{j}\right)$, which is positive by the assumption. Taking some $\varepsilon$ with $0<3 \varepsilon<\delta$ and using the assumptions (i) and (ii), we can estimate $S(r)$ as

$$
S(r) \leqq L_{0}+L_{1} \log ^{+} \rho+L_{2} \log ^{+} \frac{1}{\rho-r}+L_{3} \log ^{+} \frac{1}{r}+\varepsilon T(\rho, f)
$$

for some constants $L_{x}(0 \leqq \kappa \leqq 3)$. By virtue of Theorem 5.10 , we have only to show that $T(r, f)(f \in \mathscr{F})$ are bounded above by a constant not depending on each $f \in \mathscr{F}$. Without loss of generality, we may assume that $T(r, f) \geqq 1$ for any $r$ and $f \in \mathscr{F}$. Apply here Lemma 2.4 (ii) in [8]. For any given $r(0<r<R)$, taking some $s$ with $R-\left((R-r) / e^{2}\right)<s<R$ arbitrarily, we can find a real number $r^{\prime}$ with $r<r^{\prime}<s$ such that

$$
T\left(r^{\prime}+\frac{R-r^{\prime}}{e T\left(r^{\prime}, f\right)}, f\right) \leqq 2 T\left(r^{\prime}, f\right) .
$$

Replace $r$ and $\rho$ in (8.2) by $r^{\prime}$ and $\rho=r^{\prime}+\left(\left(R-r^{\prime}\right) / e T\left(r^{\prime}, f\right)\right)$ respectively. We have then

$$
\begin{aligned}
S\left(r^{\prime}\right) \leqq & L_{0}^{\prime}+L_{1}^{\prime} \log ^{+} r^{\prime}+L_{2}^{\prime} \log ^{+} \frac{1}{R-r^{\prime}}+L_{3}^{\prime} \log ^{+} \frac{1}{r^{\prime}} \\
& +L_{4}^{\prime} \log ^{+} T\left(r^{\prime}, f\right)+2 \varepsilon T\left(r^{\prime}, f\right)
\end{aligned}
$$

for new constants $L_{\kappa}^{\prime}(0 \leqq \kappa \leqq 4)$. On the other hand, it holds that

$$
\log ^{+} r^{\prime} \leqq \log ^{+} R, \quad \log ^{+} \frac{1}{R-r^{\prime}} \leqq \log ^{+} \frac{1}{R-s}, \quad \log ^{+} \frac{1}{r^{\prime}} \leqq \log ^{+} \frac{1}{r}
$$

and

$$
\log ^{+} T\left(r^{\prime}, f\right) \leqq \varepsilon T\left(r^{\prime}, f\right)+K(\varepsilon)
$$

for some constant $K(\varepsilon)$ depending only on $\varepsilon$. Therefore,

$$
\delta T\left(r^{\prime}, f\right) \leqq L_{0}^{\prime \prime}+3 \varepsilon T\left(r^{\prime}, f\right)
$$

for a new constant $L_{0}^{\prime \prime}$ not depending on each $f \in \mathscr{F}$. This shows that $T(r, f)\left(\leqq T\left(r^{\prime}, f\right)\right)$ are bounded above by a constant $(1 /(\delta-3 \varepsilon)) L_{0}^{\prime \prime}$ not depending on $f \in \mathscr{F}$. The proof is complete.

Remark. As is easily shown by the above proof, the assumptions (i) and (ii) of Theorem 8.1 can be replaced by the condition that

$$
K_{4} \sum_{j=1}^{q} \log ^{+}\left|\Psi\left(f, H_{j}\right)\right|+\sum_{j=N+2}^{q} \Psi\left(f, H_{j}\right)-W_{f}^{*}
$$


are uniformly bounded, where $K_{4}$ is a constant given in Theorem 6.2.

For the case $N=1$, we have more precise conclusion (c.f., [18], p. 21).

THEOREM 8.3. Let $\mathscr{F}$ be a family of meromorphic functions on $B(R)$ each of which gives a holomorphic map into $P_{1}(C)$. Suppose that there exist mutually distinct values $a_{1}, a_{2}, \cdots, a_{q}(q \geqq 3)$ such that, for any $\varphi \in \mathscr{F}$, $\varphi(z) \not \equiv a_{j}, \varphi(z)-a_{j}$ has no zeros of multiplicities $<m_{j}(1 \leqq j \leqq q)$ and

$$
\frac{1}{m_{1}}+\frac{1}{m_{2}}+\cdots+\frac{1}{m_{q}}<q-2 .
$$

Then, $\mathscr{F}$ is a normal family.

Proof. For our purpose, we may assume that $n=1$. In fact, according to T. Nishino's result ([11]), a family of meromorphic functions is normal if and only if it is normal with respect to each variable separately. On the other hand, if a holomorphic function $\varphi\left(z_{1}, \cdots, z_{n}\right)$ has a zero of multiplicity $m$ at $a=\left(a_{1}, a_{2}, \cdots, a_{n}\right)$, then $\varphi\left(z_{1}, a_{2}, \cdots, a_{n}\right)$ has a zero of multiplicity $\geqq m$ at $z_{1}=a_{1}$ as a holomorphic function of $z_{1}$ whenever $\varphi\left(z_{1}, a_{2}, \cdots, a_{n}\right) \not \equiv 0$.

We shall prove Theorem 8.3 for the case $n=1$ by the analogous argument as in the proof of Theorem 2 in [2]. The domain $B(R)$ may be assumed to be $\Delta:=B(1)=\{|z|<1\}$. For suitable homogeneous coordinates $w_{0}: w_{1}$ on $P_{1}(C)$, we can write $a_{j}^{0}: a_{j}^{1}(1 \leqq j \leqq q)$, where $a_{1}=1: 0$, $a_{2}=0: 1$ and $\left|\alpha_{j}^{0}\right|^{2}+\left|\alpha_{j}^{1}\right|^{2}=1(3 \leqq j \leqq q)$. In this case, if $\varphi(0) \neq 0, \infty$, the quantity $W_{\varphi}^{*}$ is given by $\log \left|\varphi^{\prime}(0) / \varphi(0)\right|$ and we have

$$
\Psi\left(\varphi, a_{j}\right)=\log \left|a_{j}^{0}-a_{j}^{1} \varphi(0)\right|-\log ^{+}|\varphi(0)|,
$$

particularly, $\Psi\left(\varphi, a_{1}\right)=-\log ^{+}|\varphi(0)|$ and $\Psi\left(\varphi, a_{2}\right)=\log |\varphi(0)|-\log ^{+}|\varphi(0)|$, where $\Psi\left(\varphi, a_{j}\right)=\Psi\left(\varphi, H_{j}\right)$ for $H_{j}=\left\{a_{j}^{1} w_{0}-a_{j}^{0} w_{1}=0\right\}(1 \leqq j \leqq q)$. On the other hand, as in [2], p. 236,

$$
\log |u|+A \log ^{+} \log ^{+}\left|\frac{1}{u}\right| \leqq \log ^{+}|u|+A \log A
$$

if $A>e$. Therefore, we obtain

$$
K_{4} \sum_{j=1}^{q} \log ^{+}\left|\Psi\left(\varphi, a_{j}\right)\right|+\sum_{j=3}^{q} \Psi\left(\varphi, a_{j}\right) \leqq M_{0}+M_{1}|\log | \varphi(0)||
$$

for some constants $M_{0}$ and $M_{1}$, where $K_{4}$ is a constant given in Theorem 6.2 which may be assumed to be larger than $e$. For our purpose it 
suffices to show that $\mathscr{F}$ is normal at any $z_{0} \in \Delta$. There is no loss of generality in assuming that $z_{0}$ is the origin. Take an arbitrary sequence $\left\{\varphi^{(p)}\right\}$ in $\mathscr{F}$. Firstly, we assume that there exists a positive constant $M^{*}$ such that, for some sequence $\left\{\alpha_{k}\right\}$ in $\Delta$ and a subsequence $\left\{\varphi^{\left(p_{k}\right)}\right\}$ of $\varphi^{(p)}, \lim _{k \rightarrow \infty} \alpha_{k}=0, \varphi^{\left(p_{k}\right)}\left(\alpha_{k}\right) \neq 0(=1: 0), \neq \infty(=0: 1)$ and

$$
\log \left|\frac{\varphi^{\left(p_{k}\right)}\left(\alpha_{k}\right)}{\varphi^{\left(p_{k}\right)^{\prime}}\left(\alpha_{k}\right)}\right|+M_{1}|\log | \varphi^{\left(p_{k}\right)}\left(\alpha_{k}\right)|| \leqq M_{*}
$$

where $M_{1}$ is a constant given in (8.4). As in [2], put $\varphi_{k}(z):=$ $\varphi^{\left(p_{k}\right)}(z)\left\{\left(z-\alpha_{k}\right) /\left(\bar{\alpha}_{k} z-1\right)\right\}$ and consider a family $\mathscr{F}^{\prime}:=\left\{\varphi_{k}\right\}$. Then, by Remark to Theorem 8.1 and (8.5), $\mathscr{F}^{\prime}$ is normal at the origin. So, by virtue of Lemma $1^{\prime}$ in [2], we can conclude that $\left\{\varphi^{\left(p_{k}\right)}(z)\right\}$ has a subsequence which converges compactly on a neighborhood of the origin.

Now, let us consider the case that (8.5) does not hold. Then, we can find some $r_{0}(>0)$ and $M\left(r_{0}\right)(>0)$ such that

$$
\frac{\left|\varphi^{\left(p_{k}\right)^{\prime}}(z)\right|\left|\varphi^{\left(p_{k}\right)}(z)\right|^{\ell-1}}{1+\left|\varphi^{\left(p_{k}\right)}(z)\right|^{2 \ell}} \leqq M\left(r_{0}\right)
$$

on $\left\{z:|z| \leqq r_{0}\right\}$, where $\ell$ is an arbitrarily chosen integer with $\ell \geqq M_{1}$. In fact, by the assumption, there is some $r_{0}>0$ such that

$$
\log \left|\frac{\left.\left.\varphi^{\left(p_{k}\right)}\right) z\right)}{\varphi^{\left(p_{k}\right)^{\prime}}(z)}\right|+\ell|\log | \varphi^{\left(p_{k}\right)}(z)|| \geqq 1
$$

on $\left\{z:|z| \leqq r_{0}\right\}$ for all but finitely many $\varphi^{\left(p_{k}\right)}$. Then, since

$$
\begin{aligned}
& |\log | \frac{\varphi^{\left(p_{k}\right)}(z)}{\varphi^{\left(p_{k}\right)^{\prime}}(z)}|+\ell| \log \left|\varphi^{\left(p_{k}\right)}(z)\right|\left|-\log \frac{1+\left|\varphi^{\left(p_{k}\right)}(z)\right|^{2 \ell}}{\left|\varphi^{\left(p_{k}\right)^{\prime}}(z)\right|\left|\varphi^{\left(p_{k}\right)}(z)\right|^{\ell-1}}\right| \\
& \leqq \\
& \leqq
\end{aligned}
$$

we have

$$
\log \frac{1+\left|\varphi^{\left(p_{k}\right)}(z)\right|^{2 \ell}}{\left|\varphi^{\left(p_{k}\right)^{\prime}}(z)\right|\left|\varphi^{\left(p_{k}\right)}(z)\right|^{\ell-1}} \geqq 1-\log 2(>0) .
$$

which gives (8.6). The inequality (8.6) means that $\left\{\left(\varphi^{\left(p_{k}\right)}\right)^{\ell}\right\}$ satisfies the condition in Theorem 6.3 in [8]. Consequently, $\left\{\left(\varphi^{\left(p_{k}\right)}\right)^{\ell}\right\}$ is normal. Then, $\left\{\varphi^{\left(p_{k}\right)}\right\}$ itself is obviously normal. We have thus Theorem 8.3.

We shall prove lastly a generalized Schottky-Landau type Theorem.

THEOREM 8.7. Let $H_{j}(1 \leqq j \leqq q)$ be $q(\geqq N+2)$ hyperplanes in gen- 
eral position. Suppose that there is a meromorphic map $f$ of $B(R)$ into $P_{N}(C)$ such that $f(0) \notin \bigcup_{j=1}^{q} H_{j}, W_{j}^{*} \neq-\infty$ and $\nu\left(f, H_{j}\right) \geqq m_{j}$ on $\left|\nu\left(f, H_{j}\right)\right|$ for some positive integers $m_{j}$ with $\sum_{j=1}^{q} \frac{1}{m_{j}}<\frac{q-N-1}{N}$.

Then,

$$
R \leqq R_{0}
$$

for a fixed constant $R_{0}$ depending only on $H_{j}, m_{j}(1 \leqq j \leqq q)$, the value $f(0)$ and the quantity $W_{f}^{*}$.

Proof. Assume the contrary. Then, we can find a sequence of positive numbers

$$
R_{1}<R_{2}<\cdots<R_{p}<\cdots
$$

with $\lim _{p \rightarrow \infty} R_{p}=\infty$ such that for each $p$ there exists a meromorphic maps $f^{(p)}$ of $B\left(R_{p}\right)$ into $P_{N}(C)$ with $f^{(p)}(0)=v_{0}$ and $W_{f^{(p)}}^{*}=K$ satisfying the condition that $\nu\left(f^{(p)}, H_{j}\right) \geqq m_{j}$ on $\left|\nu\left(f^{(p)}, H_{j}\right)\right|$, where a point $v_{0} \in P_{N}(C)$ - $\bigcup_{j=1}^{q} H_{j}$ and a constant $K$ are fixed. By Theorem 8.1, a sequence $\left\{f^{(p)}: p \geqq p_{0}\right\}$ is an $m$-normal family of meromorphic maps of $B\left(R_{p_{0}}\right)$ into $P_{N}(C)$ and, moreover, normal at the origin. By the diagonal argument, we can find a subsequence $\left\{f^{\left(p_{k}\right)}\right\}$ which converges compactly on a neighborhood of the origin and converges meromorphically on $B\left(R_{p}\right)$ for any $p$. The limit $f$ is a meromorphic map of $C^{n}$ into $P_{N}(C)$ which is holomorphic at the origin and satisfies the condition that $f(0)=w_{0}$, $W_{f}^{*}=K$ and $\nu\left(f, H_{j}\right) \geqq m_{j}$ on $\left|\nu\left(f, H_{j}\right)\right|$, because $\lim _{p \rightarrow \infty} \nu\left(f^{(p)}, H_{j}\right)=\nu\left(f, H_{j}\right)$. This contradicts Corollary 7.5. We have Theorem 8.7.

\section{REFERENCES}

[1] H. Cartan, Sur les zéros des combinaisons linéaires de p fonctions holomorphes données, Mathematica, 7 (1933), 5-31.

[2] D. Drasin, Normal families and the Nevanlinna theory, Acta Math., 122 (1969), 231-263.

[3] J. Dufresnoy, Théorie nouvelle des familles complexes normales; applications à l'étude des fonctions algebroïdes, Ann. E. N. S., (3) 61 (1944), 1-44.

[ 4 ] H. Fujimoto, On holomorphic maps into a taut complex space, Nagoya Math. J., 46 (1972), 49-61.

[ 5 ] H. Fujimoto, Extensions of the big Picard's theorem, Tôhoku Math. J., 24 (1972), 415-422.

[6] H. Fujimoto, Families of holomorphic maps into the projective space omitting some hyperplanes, J. Math. Soc. Japan 25 (1973), 235-249. 
[ 7 ] H. Fujimoto, On meromorphic maps into the complex projective space, J. Math. Soc. Japan, 26 (1974), 272-288.

[ 8 ] W. K. Hayman, Meromorphic functions, Clarendon Press, Oxford, 1964.

[9] P. Kiernan and S. Kobayashi, Holomorphic mappings into projective space with lacunary hyperplanes, Nagoya Math. J., 50 (1973), 199-216.

[10] P. Montel, Sur les familles quasi-normales de fonctions analytiques, Bull. Soc. Math., 52 (1924), 85-114.

[11] T. Nishino, Sur les familles de surface analytiques, J. Math. Kyoto Univ., 1 (1962), $357-377$.

[12] T. Nishino, Sur une propriété des familles de fonctions analytiques de deux variables complexes, J. Math. Kyoto Univ., 4 (1965), 255-282.

[13] H. Rutishauser, Über die Folgen und Scharen von analytischen und meromorphen Funktionen mehrerer variabeln, sowie von analytischen Abbildungen, Acta Math., $83(1950), 249-325$.

[14] W. Stoll, Die beiden Hauptsätze der Wertverteilungstheorie bei Funktionen mehrerer komplexen Veränderlichen (I), Acta Math., 90 (1953), 1-115 and (II) Acta Math., 92 (1954), 55-169.

[15] W. Stoll, The growth of the area of a transcendental analytic set I, Math. Ann., 156 (1964), 47-78 and II, Math. Ann. 156 (1964), 144-170.

[16] W. Stoll, Normal families of non-negative divisors, Math. Zeits., 84 (1964), 154218.

[17] P. Thullen, Über die wesentlichen Singularitäten analytischer Functionen und Flächen in Raum von $n$ komplexen Veränderlichen, Math. Ann., 111 (1935), 137157.

[18] G. Valiron, Familles normales et quasi-normales de fonctions meromorphes, Mém. Sci. Math., Fasc. 38, 1929.

Nagoya University 